\title{
Hypothesis of Primary Particles and the Creation of the Big Bang and Other Universes
}

\author{
Slobodan Spremo \\ Mathematical Grammar School, Belgrade, Serbia \\ Email: slobodan.spremo@gmail.com
}

How to cite this paper: Spremo, S. (2019) Hypothesis of Primary Particles and the Creation of the Big Bang and Other Universes. Journal of Modern Physics, 10, 1532-1547.

https://doi.org/10.4236/jmp.2019.1013102

Received: October 14, 2019

Accepted: November 17, 2019

Published: November 20, 2019

Copyright () 2019 by author(s) and Scientific Research Publishing Inc. This work is licensed under the Creative Commons Attribution International License (CC BY 4.0).

http://creativecommons.org/licenses/by/4.0/

\begin{abstract}
In this paper, we have presented a new approach to the dynamics of hypothetical primary particles, moving at speeds greater than the speed of light in a vacuum within their flat spacetime, which is why we understood the reason why they have not been detected so far. By introducing a new factor, we have linked the space-time coordinates of primary particles, within different inertial frames of reference. We have shown that transformations of coordinates for primary particles with respect to different inertial frames of reference, based on this factor, constitute the Lorentz transformations. Utilizing this factor, we have set the foundations of primary particle dynamics. The results obtained for the dynamic properties of these particles are in accordance with the fundamental laws of physics, and we expect them to be experimentally verifiable. Likewise, due to their dynamic properties, we have concluded that the Big Bang could have occurred during a mutual collision of the primary particles, with a sudden speed decrease of some of these particles to a speed slightly greater than the speed of light in a vacuum, which would release an enormous amount of energy. Created in such manner, our Universe would possess a limit on the maximum speed of energy-mass transfer, the speed of light in a vacuum, which we will show after introducing the dynamic properties of these particles. Similarly, we have concluded that the creation of other universes, possessing a different maximum speed of energy-mass transfer, occurred during the collision of these particles as well, only by means of deceleration of some of these particles to a speed slightly greater than the maximum speed of energy-mass transfer in that particular universe.
\end{abstract}

\section{Keywords}

Big Bang, Flat Spacetime, Lorentz Transformations

\section{Introduction}

According to the hypothesis, primary particles move at speeds $u$ much greater 
than the maximum speed of energy-mass transfer in different universes $k$, and because of this they are in their flat spacetime, wherein the aforementioned space is homogeneous and isotropic and time is homogeneous. Simultaneously, their lower border speed equals $k$, i.e. they can only move at speeds that surpass the values of it $u>k$. In our Universe, the maximum speed of energy-mass transfer is $k=c$. We have shown what the basic kinematic and dynamic properties of these hypothetical particles look like, based on the newly introduced $\xi$ factor which links space-time coordinates between different inertial frames of reference. It is widely known that our Universe originated in the Big Bang, but that modern physics cannot explain events preceding Planck time. We expect further development of this hypothesis to clarify the Big Bang itself.

In the following section of this paper, we will discuss potential kinematic and dynamic properties of hypothetical primary particles with regard to our Universe, in which the maximum speed of energy-mass transfer is the same as the speed of light in a vacuum.

The facts known thus far are the following:

- Particles that possess the rest mass can approach the speed of light by increasing their momentum and energy. Let's assume they move with $v<c$.

- The maximum speed of energy-mass transfer is the same as the speed of light in a vacuum $c$.

\section{Newly described:}

- Primary particles, whose speeds we will denote by $u$, are able to move faster than light $c<u \leq u_{p},\left(u_{p} \gg c\right)$.

The postulates on which the primary particle hypothesis is based on, extend the first two postulates of the special theory of relativity with the third:

1) The Principle of Relativity: The laws of physics are invariant in all inertial frames of reference.

2) The Principle of Constancy of the speed of light: The speed of light in a vacuum is the same for all observers, regardless of the motion of the light source or observer.

3) The speeds of the primary particles $u$, may possess values $c<u \leq u_{p}$, $\left(u_{p} \gg c\right)$, that are independent of the choice of inertial frame of reference from which observations are being made.

\section{Time Dilation for Primary Particles}

Einstein's relativity of the notion of simultaneous occurrence of some events [1] can also be extended to primary particles moving faster than light.

We will imagine how observers from the "mobile" $S$ ' and the "stationary" $S$ frames of reference see the time between two events in the world of primary particles, through an example of the time required for the primary particle to pass a certain distance from the point of departure to the primary particle reflector (Figure 1). Let us assume that $S$ ' moves in the positive direction of the $x$-axis at the speed of light $c$, relative to system $S$, because we want to find the factor 


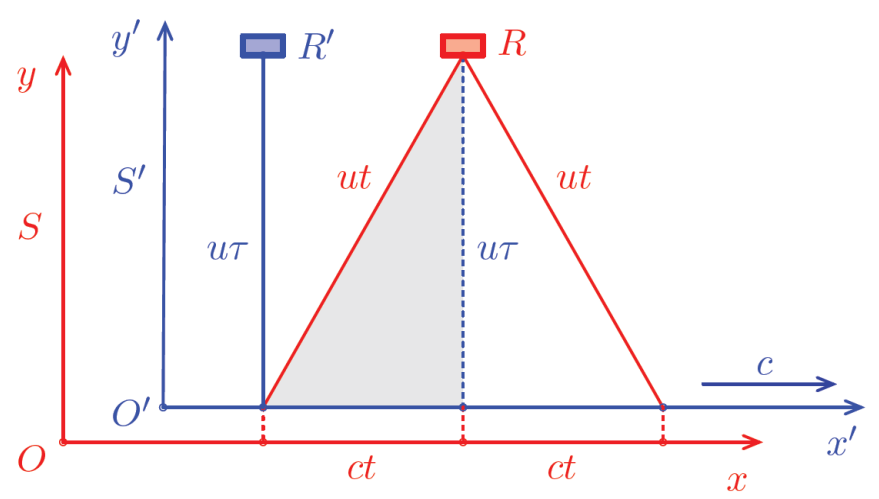

Figure 1. The observer $O$ from the $S$ perceives that the observer $O$ ' and the reflector are moving to the right at speed of light $c$, which represents the lower border velocity of the primary particles movement, hence the series of these events appear different to him. By the time primary particle reaches it, the reflector moves to the right by distance $c t$, hence the primary particle travels the distance $u t$, where $t$ represents the time required for primary particle to relocate from the point of the viewer $O$ 'to the reflector at the position $R$. After time $2 t$, the primary particle, starting from the point $O$ 'and reflecting of the reflector, returns to $O$ 'again, but observed from the $S$, by passing the distance $2 u t$. Therefore, the observer $O$ from the $S$ concludes that the primary particle will reach the reflector only if it leaves the point of the observer at some angle in relation to the vertical plane. Note also that observer $O$ must possess two synchronized clocks at the departure and arrival points of the primary particle, which are immobile in his frame of reference $S$, and compare their display with the display of a mobile clock located in the frame of reference $S$. Thus, the observer $O$ from the $S$ measures the dilated time $t$ in relation to the time $\tau$ measured by the observer $O$ 'from the $S$ '.

through which the space-time connection of possible speeds of the primary particles $u$ with $c$, would be implemented. Above the observer $O$ ' and the frame of reference $S$, a reflector of primary particles $R^{\prime}$ is located at a certain distance, perpendicular to the path of the primary particle in that frame of reference. Observer $O$ ' measures time for which the primary particle proceeding perpendicular towards the reflector returned as $2 \tau$, travels the distance $2 u \tau$. Note that in order to measure this time, the observer $O$ ' requires only one clock which is permanently situated at the same location from which the primary particle starts and to which it returns.

From the shaded triangle, we can see the following:

$$
u^{2} t^{2}=c^{2} t^{2}+u^{2} \tau^{2}, \quad t^{2}=\frac{u^{2}}{u^{2}-c^{2}} \tau^{2}, \quad t=\frac{1}{\sqrt{1-\frac{c^{2}}{u^{2}}}} \tau .
$$

We can also introduce the $\xi$ factor:

$$
\xi=\frac{1}{\sqrt{1-\frac{c^{2}}{u^{2}}}},
$$

so the following applies $t=\xi \tau$

Similarly, if these events were to play out in the $S$, in the manner that the pri- 
mary particle moved from and returned to the same place in this frame of reference, the observer $O$ 'from the $S^{\prime}$ would measure the same dilation of time, since in relation to him, the $S$ moves at speed of light $c$ only in the opposite direction, to the left. In accordance with the postulate on the independence of primary particle speed from choice of the inertial frame of reference from which it is being observed, for both observers it moves at the identical speed $u$. None of the observers $O$ and $O$ ' have the means of determining whether or not they are moving, i.e. each of them is in a state of rest in his own frame of reference. Therefore, the names of two frames of reference "mobile" and "stationary" are written in inverted commas.

The shortest possible time between two events, proper time $\tau$, is measured by the observer from whose perspective they are taking place at the same location in space. We therefore conclude that the time elapsed between the two events depends on how far they have unfolded within the two observed frame of references, i.e. that a connection between spatial and time intervals exists.

\section{The Transformation of Space-Time Coordinates for Primary Particles}

Since primary particles moving at speeds higher than the speed of light $u>c$, it is necessary to introduce new transformations of space-time coordinates between inertial frames of reference. They must apply to all speeds of these particles of $u$ which is slightly higher than $c$ to $u_{p}$. As we can see (Figure 2), the same event has different coordinates in two different inertial frames of reference.

$$
x^{\prime}=C x+D t, \quad t^{\prime}=K x+L t,
$$

where $C, D, K$ and $L$ represent constants to be determined. If we observe the movement of a primary particle along the $x$-axis, it will, from the perspective of the frame of reference $S^{\prime}$ at time moments $t_{1}^{\prime}$ and $t_{2}^{\prime}$, possess the spatial coordinates $x_{1}^{\prime}$ and $x_{2}^{\prime}$, and its shift will be

$$
\Delta x^{\prime}=x_{2}^{\prime}-x_{1}^{\prime}=C\left(x_{2}-x_{1}\right)+D\left(t_{2}-t_{1}\right)
$$

The time interval in the $S$, during which the movement occurred is

$$
\Delta t^{\prime}=t_{2}^{\prime}-t_{1}^{\prime}=K\left(x_{2}-x_{1}\right)+L\left(t_{2}-t_{1}\right) .
$$

Based on this, the velocity of the observed primary particle moving along the $x$-axis equals:

$$
u^{\prime}=\frac{\Delta x^{\prime}}{\Delta t^{\prime}}=\frac{C \Delta x+D \Delta t}{K \Delta x+L \Delta t} .
$$

Relative to the frame of reference $S$ the velocity of that same point is $u=\frac{\Delta x}{\Delta t}$, and the relation of these two velocities is given via expression

$$
u^{\prime}=\frac{C u+D}{K u+L} .
$$

In order to determine the constants $C, D, K$ and $L$ in this expression, we will consider several special cases of motion. 


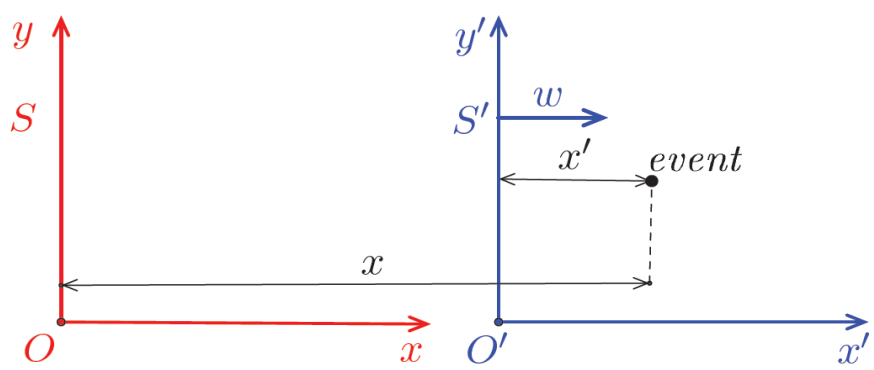

Figure 2. Let us assume the frame of reference $S$ to be "stationary" and the frame of reference $S$ ' to be "mobile", moving in the positive direction of $x$-axis at a constant velocity of $\omega \leq u_{p}$. Assume that both reference systems coincided at the moment of time $t=t^{\prime}=0$. The same event would be described by the observer from $S$ in space-time coordinates $(x, y, z, t)$, while the observer from $S^{\prime}$ would assign them the coordinates $\left(x^{\prime}, y^{\prime}, z^{\prime}, t^{\prime}\right)$. It is necessary now to find new coordinate transformations that would also apply at the higher relative velocities of frames of reference, than the speed of light. Along the $y$ and $z$ axes, no movement $S$ and $S^{\prime}$ occurs, and as in the cases of Galilean and Lorentz transformations, $y^{\prime}=y, z^{\prime}=z$, applies. In order to determine the functional connection between the remaining two coordinates, one of space and one of time, we have to acknowledge that it should preserve the properties of space and time. The basic properties of the space are homogeneity and isotropy, and the basic property of time is homogeneity, which is directly related with the conservation laws in mechanics. Therefore, the coordinate transformation law we are searching for has to be linear, hence the interconnection of the coordinates is linear.

We will first observe the border case. The primary particle is idle relative to $S$, i.e. is located in its own frame of reference, at its lowest possible velocity in relation to $S$. In this case, $u^{\prime}=0$ while $u$ has slightly greater value than $c$, i.e. $u \rightarrow c$. Therefore, observed from a "stationary" frame of reference, the primary particle moves at the same velocity as the "mobile" frame of reference. Thus, expression (6) becomes

$$
0=\frac{C c+D}{K c+L},
$$

and the following applies

$$
D=-C c .
$$

Vice versa, due to relativity of motion, when the primary particle is idle relative to $S$ i.e. $u=0$, then its velocity in relation to $S, u^{\prime} \rightarrow-c$. If we substitute this in (6) using (7) we get $-c=-\frac{C c}{L}$, i.e.

$$
C=L .
$$

We will now use the third postulate according to which the possible primary particle velocities appear identical, observed from all inertial frames of reference

$$
u=u^{\prime} \text {, }
$$

and if we substitute this in (6) using (7) and (8) we get $u=\frac{C u-C c}{K u+C}$, from which the following applies 


$$
K=-C \frac{c}{u^{2}} .
$$

Equations (2), after replacing the values $D, K$ and $L$, become

$$
x^{\prime}=C(x-c t), \quad t^{\prime}=C\left(t-\frac{c}{u^{2}} x\right),
$$

in which only the constant $C$ remains unspecified. According to the principle of relativity, a complete equality of the observed frames of reference exists. Which means that for an "stationary" frame of reference we can take $S$ ' and consider that the frame of reference $S$ moves at velocity " $-\omega$ " in relation to it. Based on that, equations that connect $x$ and $t$ with $x$ 'and $t$ 'read

$$
x=C\left(x^{\prime}+c t^{\prime}\right), \quad t=C\left(t^{\prime}+\frac{C}{u^{2}} x^{\prime}\right) .
$$

When we replace (12) in (11) we get

$$
x^{\prime}=C^{2}\left(x^{\prime}+c t^{\prime}-c t^{\prime}-\frac{c}{u^{2}} x^{\prime}\right),
$$

from which we can see that $C=\frac{1}{\sqrt{1-\frac{c^{2}}{u^{2}}}}$, i.e. $C$ have the same values as the previously introduced $\xi$ factor (1). With constants determined in such a manner, we obtain the transformations in the form

$$
x^{\prime}=\frac{x-c t}{\sqrt{1-\frac{c^{2}}{u^{2}}}}, \quad y^{\prime}=y, \quad z^{\prime}=z, \quad t^{\prime}=\frac{t-\frac{c}{u^{2}} x}{\sqrt{1-\frac{c^{2}}{u^{2}}}},
$$

i.e.

$$
x=\frac{x^{\prime}+c t^{\prime}}{\sqrt{1-\frac{c^{2}}{u^{2}}}}, \quad y=y^{\prime}, \quad z=z^{\prime}, \quad t=\frac{t^{\prime}+\frac{c}{u^{2}} x^{\prime}}{\sqrt{1-\frac{c^{2}}{u^{2}}}}
$$

These transformations connect the space-time coordinates $(x, y, z, t)$ and $\left(x^{\prime}, y^{\prime}, z^{\prime}, t^{\prime}\right)$ of the same event observed from two inertial frames of reference $S$ and $S$ 'in the case of their motion being relative along $x$-axis at a constant velocity of $u>c$.

\section{Four-Dimensional Formulation of Coordinate Transformations for Primary Particles}

Similar to Minkowski space [2], we shall introduce a real four-dimensional space in which we will present transformations of coordinates of the primary particles as transformations of coordinates of that very space. Points in that space are position vectors 


$$
\boldsymbol{x}=x^{\sigma} \boldsymbol{e}_{\sigma}=\left(\begin{array}{c}
x^{0} \\
x^{1} \\
x^{2} \\
x^{3}
\end{array}\right)=\left(\begin{array}{c}
u t \\
x \\
y \\
z
\end{array}\right)
$$

where $x^{\sigma}$ represent contravariant components of the vector $\boldsymbol{x}$ at basis

$$
\boldsymbol{e}_{0}=\left(\begin{array}{l}
1 \\
0 \\
0 \\
0
\end{array}\right), \quad \boldsymbol{e}_{1}=\left(\begin{array}{l}
0 \\
1 \\
0 \\
0
\end{array}\right), \quad \boldsymbol{e}_{2}=\left(\begin{array}{l}
0 \\
0 \\
1 \\
0
\end{array}\right), \quad \boldsymbol{e}_{3}=\left(\begin{array}{l}
0 \\
0 \\
0 \\
1
\end{array}\right) .
$$

The metric of this space is identical to the metric of Minkowski space

$$
g_{\sigma \varsigma}=\left(\begin{array}{cccc}
1 & 0 & 0 & 0 \\
0 & -1 & 0 & 0 \\
0 & 0 & -1 & 0 \\
0 & 0 & 0 & -1
\end{array}\right)
$$

This metric is used to determine the length of a vector. A square of length of the position four-vector $x$ equals

$$
\begin{aligned}
x^{2} & =x^{\mathrm{T}} g x=\left(\begin{array}{llll}
u t & x & y & z
\end{array}\right)\left(\begin{array}{cccc}
1 & 0 & 0 & 0 \\
0 & -1 & 0 & 0 \\
0 & 0 & -1 & 0 \\
0 & 0 & 0 & -1
\end{array}\right)\left(\begin{array}{c}
u t \\
x \\
y \\
z
\end{array}\right) \\
& =\left(x^{0}\right)^{2}-\left(x^{1}\right)^{2}-\left(x^{2}\right)^{2}-\left(x^{3}\right)^{2}=u^{2} t^{2}-x^{2} .
\end{aligned}
$$

Within spacetime of the primary particles, we associate each contravariant vector to covariant components using the metric

$$
A_{\sigma}=g_{\sigma \varsigma} A^{\varsigma} \text {. }
$$

Below, we will use Einstein summation convention, which implies summation when an index is repeated twice in a single term, once as upper index and once as lower index, without writing the sum sign. Therefore, the previous form is summed by the index $\varsigma$, and the following applies

$$
\begin{aligned}
& A_{0}=g_{0 \varsigma} A^{\varsigma}=g_{00} A^{0}+g_{01} A^{1}+g_{02} A^{2}+g_{03} A^{3}=A^{0}, \\
& A_{1}=g_{1 \varsigma} A^{\varsigma}=g_{10} A^{1}+g_{11} A^{1}+g_{12} A^{2}+g_{13} A^{3}=-A^{1} .
\end{aligned}
$$

In a similar way, we get $A_{2}=-A^{2}$ and $A_{3}=-A^{3}$. We see that lowering the time index does not change the sign, while lowering the spatial index $i=1,2,3$ does change the sign, so we can write

$$
A_{0}=A^{0}, \quad A_{i}=-A^{i} .
$$

The covariant components of the position vector are

$$
\begin{aligned}
& x_{0}=x^{0}=u t, \\
& x_{1}=-x^{1}=-x, \\
& x_{2}=-x^{2}=-y, \\
& x_{3}=-x^{3}=-z .
\end{aligned}
$$


An inverse metric tensor is defined by

$$
g g^{-1}=g^{-1} g=I,
$$

where $I$ represents an identity matrix. Numerically, we easily get $g^{-1}=g$. However, tensors $g^{-1}$ and $g$ have different index layout. The inverse metric allows us to transform the lower indices into the upper ones i.e. $A^{\sigma}=g^{\sigma \varsigma} A_{\varsigma}$.

The square of the length of vector $x$ is calculated via

$$
x^{2}=g_{\sigma \varsigma} x^{\sigma} x^{\varsigma}=\left(x^{0}\right)^{2}-x^{2},
$$

and for differential of the square length between the points $x$ and $x+\mathrm{d} x$ we get

$$
\mathrm{d} s^{2}=g_{\sigma \varsigma} x^{\sigma} x^{\varsigma}=u^{2}(\mathrm{~d} t)^{2}-(\mathrm{d} \boldsymbol{r})^{2} .
$$

Boost along $X$-axis for primary particles, which is similar to the Lorentz boost, we will record in the form of

$$
\begin{aligned}
& x^{\prime 0}=\xi x^{0}-v \xi x^{1} \\
& x^{\prime 1}=-v \xi x^{0}+\xi x^{1} \\
& x^{\prime 2}=x^{2} \\
& x^{\prime 3}=x^{3}
\end{aligned}
$$

where the following applies:

$$
v=\frac{c}{u}, \quad \xi=\frac{1}{\sqrt{1-\frac{c^{2}}{u^{2}}}} .
$$

In matrix form, the previous equations are

$$
\left(\begin{array}{c}
x^{\prime 0} \\
x^{\prime 1} \\
x^{\prime 2} \\
x^{\prime 3}
\end{array}\right)=\left(\begin{array}{cccc}
\xi & -v \xi & 0 & 0 \\
-v \xi & \xi & 0 & 0 \\
0 & 0 & 1 & 0 \\
0 & 0 & 0 & 1
\end{array}\right)\left(\begin{array}{c}
x^{0} \\
x^{1} \\
x^{2} \\
x^{3}
\end{array}\right)
$$

where $4 \times 4$ matrix represents the transformation matrix $\Sigma_{\varsigma}^{\sigma}$, in which the in$\operatorname{dex} \sigma$ represents the row index and index $\varsigma$ is the column index. The matrix Equation (27) in component notation is

$$
x^{\prime \sigma}=\sum_{\varsigma}^{\sigma} x^{\varsigma} .
$$

Lorentz transformations are those linear transformations of the coordinates $x^{\prime}=\Lambda x$, where $\Lambda$ represents the real matrix $4 \times 4$, which does not alter the square of length of the four-vector, i.e. the following applies to them $x^{\prime 2}=x^{2}$, which gives

$$
\Lambda^{\mathrm{T}} g \Lambda=g .
$$

Therefore, every real $4 \times 4$ matrix that satisfies the condition (29) is a Lorenz transformation. Hence, we see that our boost matrix along $x$-axis is a Lorentz transformation, and it can be easily shown that the boost matrices along $y$-axis and $z$-axis are also Lorentz transformations, as well as the three matrices of rotation of the coordinate system. 


\section{Time Dilation as a Result of a Transformation of Coordinates for Primary Particles}

We have already derived the time dilation formula for primary particles based on postulates that apply to primary particles. We will now demonstrate how it is obtained by applying the transformations of the coordinates of the primary particles. Imagine the clock being located at the coordinate start of the inertial frame of reference $S$. It will show time $S^{\prime}$ in $t^{\prime}$ and its coordinates in $S$ would be $x^{\prime}=y^{\prime}=z^{\prime}=0$. If we replace those values of time coordinates with expressions for transformation of the space-time coordinates of primary particles (15), we get the coordinates of the clock from "stationary" frame of reference $S$ in relation to which $S^{\prime}$ moves at velocity $u: x=\frac{c t^{\prime}}{\sqrt{1-\frac{c^{2}}{u^{2}}}}, y=0, \quad z=0$, since, observed from the $S$, clock moves along $x$-axis. Thus, the remaining time coordinate

$$
t=\frac{t^{\prime}}{\sqrt{1-\frac{c^{2}}{u^{2}}}} \text {. }
$$

As we have shown earlier, the time shown by the clock in frame of reference $S$, in relation to which it remains immobile, equals less than the time measured in frame of reference $S$, that is, in this case, time $t$ 'represents own time.

\section{The Velocity-Addition Formula for Primary Particles}

We will continue to observe that $S$ as a "stationary" frame of reference in relation to which $S$ ' moves at velocity $\boldsymbol{u}=\left(u_{x}, u_{y}, u_{z}\right)$. Primary particle with the velocity of $\boldsymbol{w}=\left(w_{x}, w_{y}, w_{z}\right)$ in relation to $S$, possess the velocity $\boldsymbol{w}^{\prime}=\left(w_{x}^{\prime}, w_{y}^{\prime}, w_{z}^{\prime}\right)$ in relation to $S$, while its $x$ component

$$
w_{x}^{\prime}=\frac{\mathrm{d} x^{\prime}}{\mathrm{d} t^{\prime}} .
$$

According to the Equation (14), the differential $\mathrm{d} x^{\prime}$ and the differential $\mathrm{d} t^{\prime}$ are

$$
\mathrm{d} x^{\prime}=\frac{\mathrm{d} x-c \mathrm{~d} t}{\sqrt{1-\frac{c^{2}}{u^{2}}}}, \quad \mathrm{~d} t^{\prime}=\frac{\mathrm{d} t-\frac{c}{u^{2}} \mathrm{~d} x}{\sqrt{1-\frac{c^{2}}{u^{2}}}},
$$

while the velocity represents their ratio

$$
w_{x}^{\prime}=\frac{\frac{\mathrm{d} x}{\mathrm{~d} t}-c}{1-\frac{c}{u^{2}} \frac{\mathrm{d} x}{\mathrm{~d} t}} .
$$

Since $w_{x}=\frac{\mathrm{d} x}{\mathrm{~d} t}, \quad x$ component of velocity relative to $S$, we have 


$$
w_{x}^{\prime}=\frac{w_{x}-c}{1-\frac{w_{x} C}{u^{2}}}
$$

Correspondingly, starting from $w_{y}^{\prime}=\frac{\mathrm{d} y^{\prime}}{\mathrm{d} t^{\prime}}$ and $w_{z}^{\prime}=\frac{\mathrm{d} z^{\prime}}{\mathrm{d} t^{\prime}}$, for the remaining two components of the velocity we get

$$
w_{y}^{\prime}=\frac{w_{y} \sqrt{1-\frac{c^{2}}{u^{2}}}}{1-\frac{w_{x} c}{u^{2}}}, \quad w_{z}^{\prime}=\frac{w_{y} \sqrt{1-\frac{c^{2}}{u^{2}}}}{1-\frac{w_{x} c}{u^{2}}} .
$$

In the border case $w_{x}=u$ formula (31) becomes

$$
w_{x}^{\prime}=\frac{u-c}{1-\frac{u c}{u^{2}}}=\frac{u\left(1-\frac{c}{u}\right)}{1-\frac{c}{u}}=u .
$$

From this we can conclude that if a primary particle moves at a speed of $u$ relative to an observer from the frame of reference $S$, then, regardless of the relative speed of the system, the primary particle possesses the same speed in relation to the observer from the frame of reference $S$ ' as well. We introduced this within the third postulate.

Formulas (31) and (32) allow us to determine the components of velocity in relation to $S$ ' for known velocity components in relation to $S$. Vice versa, the following applies

$$
\begin{gathered}
w_{x}=\frac{w_{x}^{\prime}+c}{1+\frac{w_{x}^{\prime} c}{u^{2}}}, \\
w_{y}=\frac{w_{y}^{\prime} \sqrt{1-\frac{c^{2}}{u^{2}}}}{1+\frac{w_{x}^{\prime} c}{u^{2}}}, \quad w_{z}=\frac{w_{z}^{\prime} \sqrt{1-\frac{c^{2}}{u^{2}}}}{1+\frac{w_{x}^{\prime} c}{u^{2}}} .
\end{gathered}
$$

\section{Acceleration with Regard to the Primary Particle Hypothesis}

Descartes' components of acceleration of the primary particle in inertial frame of reference $S$ are defined as

$$
a_{x}=\frac{\mathrm{d} w_{x}}{\mathrm{~d} t}, \quad a_{y}=\frac{\mathrm{d} w_{y}}{\mathrm{~d} t}, \quad a_{z}=\frac{\mathrm{d} w_{z}}{\mathrm{~d} t},
$$

and in $S^{\prime}$ are

$$
a_{x}^{\prime}=\frac{\mathrm{d} w_{x}^{\prime}}{\mathrm{d} t^{\prime}}, \quad a_{y}^{\prime}=\frac{\mathrm{d} w_{y}^{\prime}}{\mathrm{d} t^{\prime}}, \quad a_{z}^{\prime}=\frac{\mathrm{d} w_{z}^{\prime}}{\mathrm{d} t^{\prime}} .
$$

From (31) we obtain

$$
\mathrm{d} w_{x}^{\prime}=\frac{\mathrm{d} w_{x}}{\left(1-\frac{w_{x} c}{u^{2}}\right)^{2}}\left(1-\frac{c^{2}}{u^{2}}\right)
$$


The rate of change of the $x$ velocity component in $S^{\prime}$ is given via relation (30) we have

$$
a_{x}^{\prime}=\frac{\mathrm{d} w_{x}^{\prime}}{\mathrm{d} t^{\prime}}=\frac{\left(1-\frac{c^{2}}{u^{2}}\right)^{\frac{3}{2}}}{\left(1-\frac{w_{x} C}{u^{2}}\right)^{3}} a_{x} .
$$

For the remaining two acceleration components using (32), an analogous method produces

$$
\begin{aligned}
& a_{y}^{\prime}=\frac{\mathrm{d} w_{y}^{\prime}}{\mathrm{d} t^{\prime}}=\frac{1-\frac{c^{2}}{u^{2}}}{\left(1-\frac{w_{x} c}{u^{2}}\right)^{2}} a_{y}+\frac{w_{y} c\left(1-\frac{c^{2}}{u^{2}}\right)}{u^{2}\left(1-\frac{w_{x} c}{u^{2}}\right)^{3}} a_{x}, \\
& a_{z}^{\prime}=\frac{\mathrm{d} w_{z}^{\prime}}{\mathrm{d} t^{\prime}}=\frac{1-\frac{c^{2}}{u^{2}}}{\left(1-\frac{w_{x} c}{u^{2}}\right)^{2}} a_{z}+\frac{w_{z} c\left(1-\frac{c^{2}}{u^{2}}\right)}{u^{2}\left(1-\frac{w_{x} c}{u^{2}}\right)^{3}} a_{x} .
\end{aligned}
$$

Based on these relations, which enable transformation of the acceleration components between the two inertial frames of reference, we can conclude that acceleration is not invariant, similar to that in the special theory of relativity. However, all inertial observers will agree that acceleration exists. Namely, if a primary particle observed from an inertial frame of reference is moving rapidly, then it is moving rapidly in all other inertial frames of reference as well. Similarly, if the acceleration in one of those inertial frames of reference equals zero, then it equals zero in all other inertial frames of reference.

\section{Fundamentals of Primary Particle Dynamics}

While moving at maximum speeds $u_{p} \gg c$, energy $E_{p}$ of these particles would match the fundamental rest energy in relativistic physics

$$
E_{p}=m_{p} c^{2},
$$

where $m_{p}$ represents the attributed mass of the primary particle.

Similar to the total energy of a relativistic particle noted via Lorentz $\gamma$ factor, we can use the $\xi$ factor for notation of the total energy of a primary particle $E_{t}$ as

$$
E_{t}=\xi m_{p} c^{2}=\frac{m_{p} c^{2}}{\sqrt{1-\frac{c^{2}}{u^{2}}}} .
$$

From (41) we can observe that the total energy of the primary particles behaves depending on their speed as in Figure 3. They possess the lowest energy $E_{t}=m_{p} c^{2}$. while moving at maximum speed $u_{p} \gg c$, and the highest when $u \rightarrow c$. 


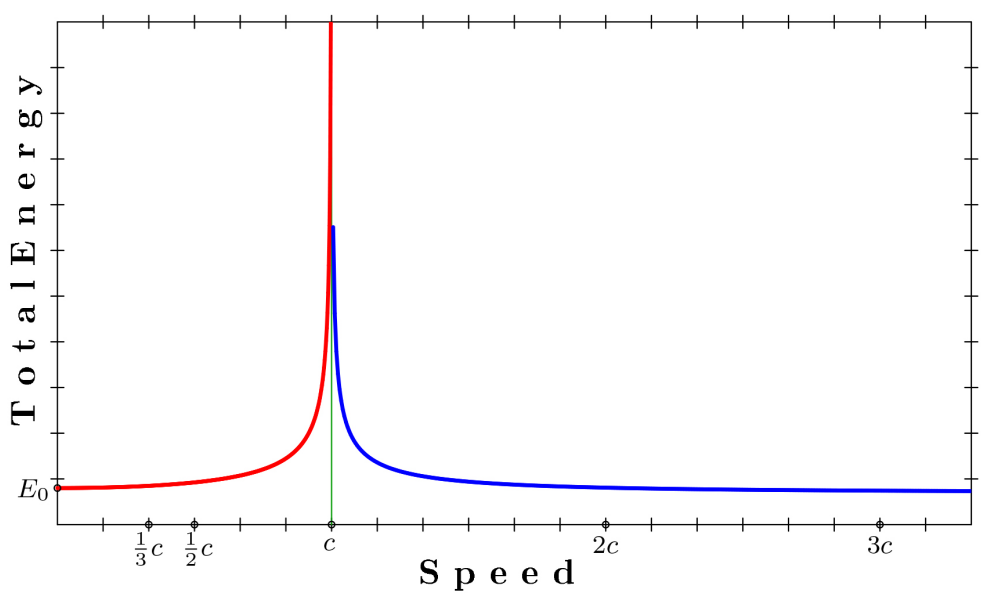

Figure 3. The dependence of total relativistic energy of the particle on speed $\mathrm{v}$ (red curve), the speed of light in a vacuum c (green line) and dependence total energy of the primary particle on speed $u$; according to the primary particles hypothesis (blue curve).

We will introduce homeokinetic energy $H$.

Conversely with kinetic energy increment that accompanies increase of the speed of particles possessing rest mass in classical and relativistic physics, homeokinetic energy of primary particles increases as they decelerate during mutual collisions, consequently increasing their total energy $E_{t}$.

$$
E_{t}=E_{p}+H \text {. }
$$

It is obvious that these particles would not interact with matter via four known interactions due to the speed of their motion $u>c$, so primary particles therefore exist in their flat spacetime, while the non-interaction of these particles with matter is the reason why they have not been detected so far. So, in order for these particles to mutually interact, a need for a new kind of interaction expanding faster than the speed of light arises.

For homeokinetic energy, from (40), (41) and (42) we get

$$
H=E_{t}-E_{p}=m_{p} c^{2}\left(\frac{1}{\sqrt{1-\frac{c^{2}}{u^{2}}}}-1\right)=m_{p} c^{2}(\xi-1) .
$$

From the energy-momentum relation $E^{2}-E_{0}^{2}=p^{2} c^{2}$, which is fundamental, similarly, for primary particles we have

$$
E_{t}^{2}-E_{p}^{2}=p_{p}^{2} c^{2},
$$

where $p_{p}$ is the momentum of a primary particle. Here, considering (40) and (41), we see

$$
m_{p}^{2} c^{4}\left(\frac{1}{\frac{u^{2}-c^{2}}{u^{2}}}-1\right)=\frac{m_{p}^{2} c^{4}-m_{p}^{2} c^{4} \frac{u^{2}-c^{2}}{u^{2}}}{\frac{u^{2}-c^{2}}{u^{2}}}=\frac{m_{p}^{2} \frac{c^{6}}{u^{2}}}{\frac{u^{2}-c^{2}}{u^{2}}}=p_{p}^{2} c^{2},
$$


from which we obtain the formula for the magnitude of the momentum a primary particle, differently defined compared to classical and relativistic physics (Figure 4)

$$
p_{p}=\frac{m_{p} c^{2}}{u \sqrt{1-\frac{c^{2}}{u^{2}}}},
$$

so that:

$$
p_{p}=\frac{E_{t}}{u} .
$$

Also, the formula for the magnitude of the momentum a primary particle (46) can be obtained in a different, more intuitive way. In relativistic physics, the magnitude of the momentum a photon is $p=\frac{E}{C}$, where the speed of light in a vacuum $c$ is independent of the choice of inertial frame of reference from which observations are being made. Similarly, the speeds of the primary particles $u$ are also independent of the choice of inertial frame of reference from which observations are being made, hence the formula for the magnitude of the momentum a primary particle is $p_{p}=\frac{E_{t}}{u}$.

For $u \gg c$ the following applies: $p_{p} \rightarrow 0$.

We will further show that the basic dynamic laws of physics apply to this form of total energy of primary particles.

We will now determine the work $W$ performed by the force $F$, directed along the $x$-axis, during deceleration of the primary particle relocating from a certain location determined by the parameters $\left(x_{1}, t_{1}, u_{p}\right)$, to the second location determined by the parameters $\left(x_{2}, t_{2}, u\right) . u_{p}$ is the maximum speed of the primary particle $\left(u_{p} \gg c\right)$. Using the definition of work and Newton's second law, which applies in the same form to primary particles as well, in accordance with the first postulate

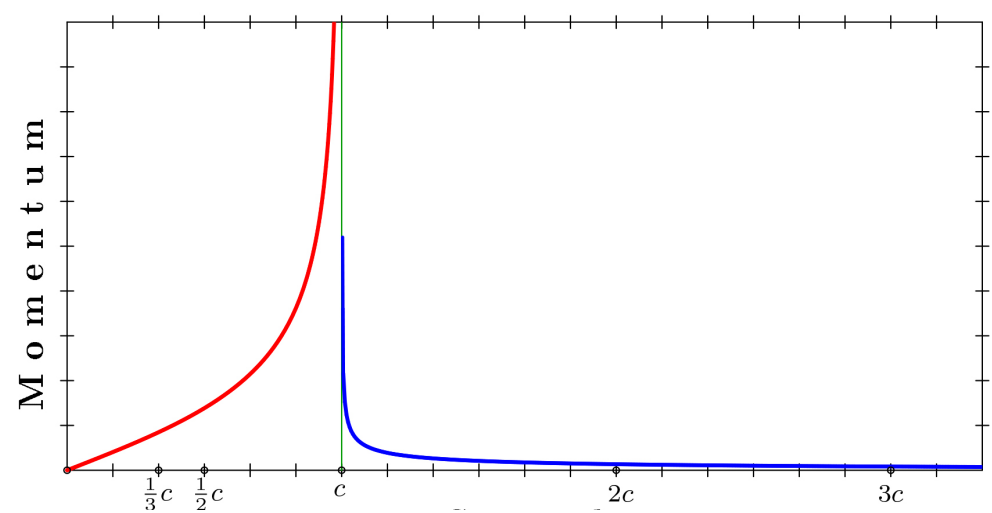

Figure 4. Dependence of relativistic momentum of the particle on speed $\mathrm{v}$ (red curve), the speed of light in a vacuum c (green line) and dependence of momentum of the primary particle on speed $u$; according to the primary particles hypothesis (blue curve). 


$$
W=\int_{x_{1}}^{x_{2}} F \mathrm{~d} x=\int_{x_{1}}^{x_{2}} \frac{\mathrm{d} p_{p}}{\mathrm{~d} t} \mathrm{~d} x
$$

and considering it is: $\mathrm{d} x=u \mathrm{~d} t$, and that the rate of change of the momentum of the primary particle is

$$
\begin{aligned}
& \frac{\mathrm{d} p_{p}}{\mathrm{~d} t}=-\frac{m_{p} c^{2}}{2}\left(u^{2}-c^{2}\right)^{-\frac{3}{2}} 2 u \frac{\mathrm{d} u}{\mathrm{~d} t}=-\frac{m_{p} c^{2} u \frac{\mathrm{d} u}{\mathrm{~d} t}}{\left(u^{2}-c^{2}\right)^{\frac{3}{2}}} \\
& \begin{aligned}
W & =-\int_{t_{1}}^{t_{2}} \frac{m_{p} c^{2} u \frac{\mathrm{d} u}{\mathrm{~d} t}}{\left(u^{2}-c^{2}\right)^{\frac{3}{2}}} u \mathrm{~d} t=-m_{p} c^{2} \int_{u_{p}}^{u} \frac{u^{2} \mathrm{~d} u}{\left(u^{2}-c^{2}\right)^{\frac{3}{2}}} \\
& =-m_{p} c^{2}\left(\int_{u_{p}}^{u} \frac{\mathrm{d} u}{\left(u^{2}-c^{2}\right)^{\frac{1}{2}}}+c^{2} \int_{u_{p}}^{u} \frac{\mathrm{d} u}{\left(u^{2}-c^{2}\right)^{\frac{3}{2}}}\right) \\
& =\left.m_{p} c^{2}\left(\frac{u}{\sqrt{u^{2}-c^{2}}}-\ln \left(u+\sqrt{u^{2}-c^{2}}\right)\right)\right|_{u_{p}} ^{u},
\end{aligned}
\end{aligned}
$$

we get the following value for work

$$
W=m_{p} c^{2}\left(\frac{u}{\sqrt{u^{2}-c^{2}}}-\frac{u_{p}}{\sqrt{u_{p}^{2}-c^{2}}}-\ln \frac{u+\sqrt{u^{2}-c^{2}}}{u_{p}+\sqrt{u_{p}^{2}-c^{2}}}\right) .
$$

For a slight decrease of the primary particle speed we see that

$$
\frac{u+\sqrt{u^{2}-c^{2}}}{u_{p}+\sqrt{u_{p}^{2}-c^{2}}} \rightarrow 1 \text {, i.e. } \ln \frac{u+\sqrt{u^{2}-c^{2}}}{u_{p}+\sqrt{u_{p}^{2}-c^{2}}} \rightarrow 0 \text {; and } \frac{u_{p}}{\sqrt{u_{p}^{2}-c^{2}}} \rightarrow 1 \text {, }
$$

because it is $u_{p} \gg c$.

Finally, for work, we get the following

$$
W=m_{p} c^{2}\left(\frac{u}{\sqrt{u^{2}-c^{2}}}-1\right)=m_{p} c^{2}\left(\frac{1}{\sqrt{1-\frac{c^{2}}{u^{2}}}}-1\right)=m_{p} c^{2}(\xi-1) .
$$

Work demonstrates how much the energy of the primary particle has changed, i.e. corresponds to its homeokinetic energy, as in Equation (43).

The relation between $p_{p}$ and $H$ is obtained by replacing (42) with (44) as

$$
p_{p}=\frac{1}{c} \sqrt{H\left(H+2 E_{p}\right)} .
$$

Also, from (40) and (44) we get

$$
E_{t}=\sqrt{p_{p}^{2} c^{2}+m_{p}^{2} c^{4}}
$$

which for $u \gg c$, i.e. $p_{p}^{2} \ll m_{p}^{2} c^{2}$ shows that $E_{t}=E_{p}=m_{p} c^{2}$.

However, in the case of $u \rightarrow c$, i.e. $p_{p}^{2} \gg m_{p}^{2} c^{2}$, we get that $E_{t}=p_{p} c$, or 


$$
p_{p}=\frac{E_{t}}{C},
$$

as we get from (46). The Equation (50) is valid for a certain high energy state of the primary particles and corresponds precisely to the relation between the momentum and the electromagnetic radiation energy which is obtained in the ultrarelativistic case for the Einstein equation $E=\sqrt{p^{2} c^{2}+m^{2} c^{4}}$.

\section{The Possibility of Interpreting the Creation of the Big Bang and Other Universes through the Collision of Primary Particles}

The Big Bang represents the very event of creating of our spacetime, containing our matter and energy. The very moment in which this event took place, as well as the short period preceding Planck time, following the occurrence of this event, are still beyond the reach of science. Namely, Einstein's general theory of relativity does not work for systems smaller than Planck length, nor for the events lasting shorter than Planck time. By expectations of modern physics, this limit should be dismantled by the quantum theory of gravity, hence, this initial period of our Universe is referred to as "the quantum gravity era". We expect this period of the Universe to be explained by the primary particle hypothesis.

According to hypothesis, primary particles moving at speeds $u \gg c$ are in their flat spacetime. Simultaneously, their total energy (41) and momentum (45) are small. However, possibility exists that during collision of such particles, which would cause the speed of some primary particle to $u \rightarrow c$, an enormous amount energy is released from the point of collision according to the relation (41), i.e. the Big Bang would ensue. Because of the lower border speed of the primary particle at the Big Bang thus attained, the speed of energy-mass transfer in our Universe would have the value $c$, which is the same from the moment of the initial singularity. The energy thus released would be the very energy generated in the Big Bang in accordance with the conservation laws in physics, resulting in creation of our spacetime, containing our matter and energy.

Due to the huge number of possible lower border speeds $k$, to which the primary particles would slow down when creating other universes, their limitation of maximum speed of energy-mass transfer would be equal to instead of $c$, to some different $k$. It is clear that this would differentiate the values of other fundamental physical constants in different universes, as well. All relations stated in the paper would be valid in other universes as well, provided $c$ is replaced with $k$ from another universe.

\section{Result}

We assumed the existence of primary particles moving within their flat spacetime at a speed $u$, greater than the maximum speed of energy-mass transfer $k$ in various universes, which, at the same time, represents their lower border speed. In addition to two postulates of the special theory of relativity, we introduced the 
third postulate as well, according to which the values of the speeds of these particles are independent of the choice of inertial frames of reference from which they are being observed. In a thought experiment, we realize that dilation of time is the greatest while these particles are moving at speeds close to lower border speed, i.e. the speed of light $c$ within our Universe, and get a $\xi$ factor that corresponds to Lorentz $\gamma$ factor. The same value of $\xi$ factor was obtained via transformations of space-time coordinates for primary particles. We have shown that these transformations are Lorentz transformations. Based on the $\xi$ factor, we have developed the dynamics of particles moving faster than light. We have proved that the assumed properties of these particles satisfy the fundamental laws of physics, which we have postulated. We recognized that the explanation of the Big Bang could be made from the standpoint of primary particles speed reduction during their mutual collision, during which the energy of the Big Bang would be released, simultaneously limiting the maximum speed of energy-mass transfer in our Universe to speed reached in that collision, $c$. The creation of other universes could be explained in a similar manner. Hence, the limitation to maximum speed of energy-mass transfer within them, as well as reached speed reduction of primary particles during big bangs in which those universes were created, would instead of $c$ have various values $k$.

\section{The Need for Development of a Primary Particle Hypothesis and Their Experimental Proof}

It is clear that in this paper we have only made a logical assumption about some properties of primary particles. Thus, we believe their further study may lead to a major shift in physics, as well as our philosophical view of the world.

Based on the described properties of primary particles, we expect their indirect experimental proof to be possible through successful explanation of the Big Bang, via further scientific work on the hypothesis of primary particles.

\section{Conflicts of Interest}

The author declares no conflicts of interest regarding the publication of this paper.

\section{References}

[1] Morris, D. (2016) The Special Theory of Relativity An Introduction: Essentials of Physics, Dulles, Virginia.

[2] Petkov, V. (2010) Minkowski Spacetime: A Hundred Years Later. Springer, New York. https://doi.org/10.1007/978-90-481-3475-5 